\title{
Hydration Biomarkers Are Related to the Differential Abundance of Fecal Microbiota and Plasma Lipopolysaccharide-Binding Protein in Adults
}

\author{
Nathaniel B. Willis ${ }^{a} \quad$ Colleen X. Muñoz ${ }^{b} \quad$ Annemarie R. Mysonhimer ${ }^{c}$ \\ Caitlyn G. Edwards ${ }^{d}$ Patricia G. Wolfe Charles H. Hillman ${ }^{\mathrm{f} g}$ \\ Nicholas A. Burd ${ }^{a, h}$ Hannah D. Holscher ${ }^{a, c, h}$ Naiman A. Khan ${ }^{a, h, i}$ \\ aDivision of Nutritional Science, University of Illinois at Urbana-Champaign, Champaign, IL, USA; ${ }^{\circ}$ Department \\ of Health Sciences, University of Hartford, West Hartford, CT, USA; 'Department of Food Science and Human

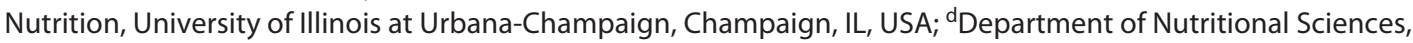

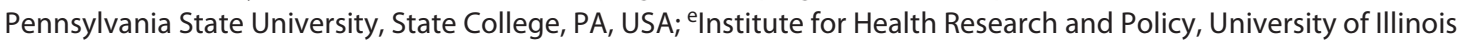

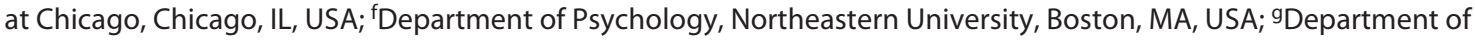 \\ Physical Therapy, Movement, \& Rehabilitation Sciences, Northeastern University, Boston, MA, USA; 'hepartment of \\ Kinesiology and Community Health, University of Illinois at Urbana-Champaign, Champaign, IL, USA; ${ }^{\text {Neuroscience }}$ \\ Program, University of Illinois at Urbana-Champaign, Champaign, IL, USA
}

\section{Keywords}

Copeptin · Vasopressin · Gastrointestinal microbiota ·

Hydration - Water · Obesity

\begin{abstract}
Introduction: Prevalence of chronic hypohydration remains elevated among adults in the USA; however, the health effects of hypohydration in regards to human gut health have not been explored. Methods: This study examined the relationship between total water intake, hydration biomarkers (first-morning urine specific gravity $\left[\mathrm{FMU}_{\mathrm{sg}}\right]$, first-morning urine volume $\left[\mathrm{FMU}_{\mathrm{vol}}\right]$, and plasma copeptin), fecal microbiota, and plasma lipopolysaccharide-binding protein (LBP) in adults (25-45 years, 64\% female). Fecal microbiota composition was assessed using 16S rRNA gene sequencing (V4 region). Immunoassays quantified plasma copeptin and LBP in fasted venous blood samples. Dietary variables were measured using 7-day food records. Linear discriminant analysis
\end{abstract}

karger@karger.com www.karger.com/anm

Karger $\stackrel{\text { ' }}{5}$
(C) 2022 The Author(s)

Published by S. Karger AG, Basel

This article is licensed under the Creative Commons Attribution 4.0 International License (CC BY) (http://www.karger.com/Services/ OpenAccessLicense). Usage, derivative works and distribution are permitted provided that proper credit is given to the author and the original publisher. effect size (LEfSe) analyzed differentially abundant microbiota based on median cutoffs for hydration markers. Multiple linear regressions examined the relationship between LBP and copeptin. Results: LEfSe identified 6 common taxa at the genus or species level that were differentially abundant in $\mathrm{FMU}_{\mathrm{sg}}$, total water (g/day), or plasma copeptin $(\mu \mathrm{g} / \mathrm{mL})$ groups when split by their median values. Uncultured species in the Bacteroides, Desulfovibrio, Roseburia, Peptococcus, and Akkermansia genera were more abundant in groups that might indicate poorer hydration status. Multivariate linear analyses revealed a positive relationship between plasma copeptin and LBP when controlling confounding variables $\left(F(6,52)=4.45, p=0.002, R^{2}=0.34\right)$. Conclusions: Taxa common between markers are associated with the intestinal mucus layer, which suggests a potential link between hydration status and intestinal mucus homeostasis. The relationship between LBP and copeptin indicates that copeptin may be sensitive to metabolic endotoxemia and potentially gut barrier function.

(c) 2022 The Author(s)

Published by S. Karger AG, Basel
Correspondence to:

Naiman A. Khan, nakhan2@illinois.edu 


\section{Introduction}

Water is one of the most important but often overlooked essential nutrients for humans [1,2]. As an integral component of all living cells, water is the single largest component of the human body and accounts for over $60 \%$ of adult body weight [3]. However, up to $65 \%$ of adults are chronically under-hydrated due to low daily water intake [4]. A $2 \%$ body weight loss from water depletion can contribute to cognitive and physical deficiencies and has been associated with obesity and chronic disease in adults [5].

Several plasma and urinary markers are sensitive to variable water intake. Body water balance is regulated by arginine vasopressin (AVP), which rises in circulation during water deprivation and preserves blood osmolality and volume by increasing renal water reabsorption. Pooled urine collected over a $24 \mathrm{~h}$ period is the ideal marker for measurement of daily hydration status, accounting for the diurnal variability of AVP [6]; however, spot urine samples, including first-morning urine (FMU), are also sensitive to variable water consumption [7]. As part of the AVP prohormone, copeptin is secreted in equimolar concentrations and can be used as a surrogate marker for AVP [8]. Beyond its role in hydration signaling, AVP is known to stimulate glycogenolysis [9], and influence gastrointestinal motility [10], while copeptin is sensitive to infection [11], and has been linked to elements of metabolic syndrome [12]. This indicates the diagnostic potential of copeptin as a marker of cardio-metabolic stress.

Lipopolysaccharide (LPS), a component of gram-negative bacteria, induces metabolic stress and systemic inflammation [13], and like copeptin, the concentration of plasma LPS has been linked to elements of metabolic syndrome [14]. However, to our knowledge, the concentration of plasma LPS or the LPS binding protein (LBP) [15], in the context of hydration status remains a novel inquiry. In fact, the effect of hydration status on gut health and relative abundance of gastrointestinal microbiota is largely unexplored.

Considering that hydration markers, LPS, and the gastrointestinal microbiota have all been implicated in metabolic regulation; this study aimed to investigate hydration biomarkers in relation to the relative abundances of fecal microbiota and plasma LBP. We hypothesized that we would observe fecal microbiota that would be differentially abundant across hydration markers (total water, first-morning urine specific gravity $\left[\mathrm{FMU}_{\mathrm{sg}}\right], \mathrm{FMU}_{\mathrm{vol}}$, and plasma copeptin). Additionally, we hypothesized that there would be a statistically significant relationship between plasma concentrations of LBP and copeptin.

\section{Materials and Methods}

\section{Participants and Study Protocol}

Participants were excluded from this study based on pregnancy or lactation, history of metabolic or neurological disease, and food allergies or intolerances. Participants were included in the primary analyses if they provided FMU samples, dietary intake information, and fecal samples $(n=156)$. This sample was then analyzed for normally distributed variables and outliers $>3 \mathrm{SD}$ from the mean were omitted $(n=10)$ resulting in a final sample size of 146 . A subsample of participants provided fasted venous blood samples that were assayed for plasma copeptin $(n=85)$ and plasma LBP $(n=95) ; 59$ samples were assayed for both markers (Fig. 1).

\section{Plasma Copeptin and LBP Analysis}

Blood was drawn from the antecubital vein following a 10-h overnight fast (with ad libitum water intake permitted), centrifuged, and stored at $-80^{\circ} \mathrm{C}$ until later analyses. Plasma biomarkers were assessed in $\mathrm{K}_{2}$ EDTA treated plasma with commercial EIA kits for copeptin (Copeptin Kit No: EK-065-32; Phoenix Pharmaceuticals, Inc. Burlingame, CA, USA), and LBP (LBP: Hycult Biotech; HK315) in duplicate and according to manufacturer instructions. Samples with intra-assay coefficients of variation $>20 \%$ were omitted from the analysis.

\section{Urine Biomarker Analyses}

FMUs were provided by participants, and all analyses were performed on fresh, nonfrozen samples. First-morning urine volume $\left(\mathrm{FMU}_{\mathrm{vol}}\right)$ was measured in a graduated beaker, and $\mathrm{FMU}_{\mathrm{sg}}$ was assessed using a digital handheld pen refractometer (ATAGO Co., Tokyo, Japan).

\section{Fecal Microbiota Analyses}

Participants provided a fresh fecal sample within 15 min of defecation. Samples were homogenized, flash-frozen, and stored at $-80^{\circ} \mathrm{C}$ until analysis. Following fecal DNA extraction utilizing the PowerLyzer PowerSoil DNA Isolation Kit (MO BIO Laboratories Inc., Carlsbad, CA, USA), the V4 region of the 16S rRNA gene was amplified on a Fluidigm Access Array. Sequencing was performed on an Illumina MiSeq or HiSeq (Illumina Inc., San Diego, CA, USA) at the W.M. Keck Center for Biotechnology, University of Illinois at Urbana-Champaign. Sequence data were analyzed with DADA2 [16] and QIIME 2 [17]. Quality score was screened at a threshold of 20 and taxonomy was assigned to the amplicon sequence variants (ASV) with the SILVA 132 reference database. Fecal microbiota diversity analyses were conducted using $\mathrm{R}$ version 4.0.0 and Phyloseq package v1.16.2 [18].

\section{Dietary Intake}

Participants recorded food and beverage intake in a 7-day food diary. Laboratory staff under the supervision of a registered dietitian entered the food records into the Nutrition Data System for Research Version 2015 (Nutrition Coordinating Center, University of Minnesota) software. Diet records were entered, separately checked for quality, and any discrepancies were resolved by a third party inspecting the original record. Mean values for total water (comprising all dietary water from food and beverage sources), total dietary fiber, and total energy intake were extracted. Normalized dietary fiber was calculated as total dietary fiber per 1,000 kcal.
Willis/Muñoz/Mysonhimer/Edwards/ Wolf/Hillman/Burd/Holscher/Khan 


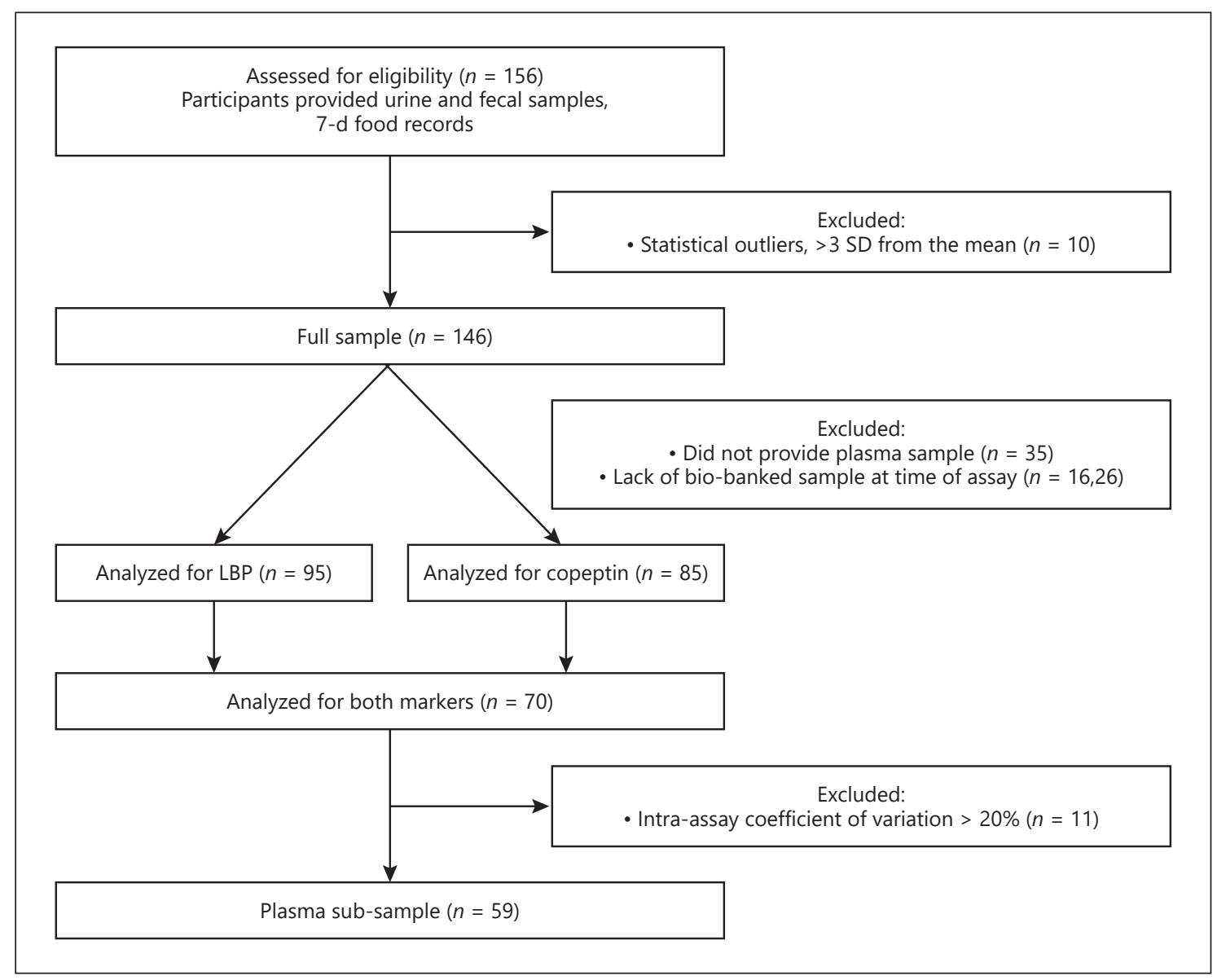

Fig. 1. Sample inclusion and analysis flowchart.

\section{Anthropometrics}

Height and weight were measured in triplicate and averaged to calculate body mass index (BMI). A stadiometer (model 240; SECA, Hamburg, Germany) and a digital scale (WB-300 Plus; Tanita, Tokyo, Japan) were used to measure height and weight, respectively.

\section{Statistical Approach}

Variables of interest were inspected for normality and a natural $\log$ transformation was used for the non-normally distributed variables to be included in linear analyses. Two-tailed Pearson correlations were conducted amongst variables including age, sex, $\mathrm{BMI}$, total water, $\mathrm{FMU}_{\mathrm{sg}}, \mathrm{FMU}_{\mathrm{vol}}$, copeptin, normalized dietary fiber, and Firmicutes to Bacteroides ratio (F:B). A Pearson partial correlation was then conducted to adjust for age, sex, BMI in this analysis. Hydration variables (total water, $\mathrm{FMU}_{\mathrm{sg}}$, FMU vol, and copeptin) were then split by their respective medians to examine above/below median group differences in demographic variables via Student's $t$ test, and in the relative abundance of microbiota between groups via linear discriminant analysis effect size (LEfSe).

Alpha diversity was measured via pairwise comparisons using the Wilcoxon rank-sum test with continuity correction and the Holm $p$ value adjustment method. Beta diversity was measured with principal coordinate analysis and permutational multivariate analysis of variance. Microbiota taxa summaries were formatted for input into LEfSe (Huttenhower Lab Galaxy Server) and analyzed for differential abundance based on above and below median groups for hydration markers of interest. Differentially abundant taxa were ranked by LEfSe; those with a Kruskal-Wallis threshold below $\alpha=0.05$ and an LDA log score of at least \pm 2 were visualized in plots. LEfSe was conducted on both the full sample $(n=146)$ and the copeptin subsample $(n=85)$. Finally, multiple linear regression was conducted to explain variability in plasma copeptin by LBP in a model controlling for age, sex, BMI, normalized dietary fiber, and total water/day $(n=59)$.

\section{Results}

The full sample was split separately by the median total water (2,438 g/day), FMU sg $_{\text {(1.018), and FMU }}$ vol $(258 \mathrm{~mL})$ to observe differences between groups (Table 1). Copeptin median $(1.14 \mathrm{ng} / \mathrm{mL})$ group comparison was also observed for subjects with plasma samples. Above/below 
Table 1. Sample descriptive data with mean and standard deviation values for variables of interest

\begin{tabular}{|c|c|c|}
\hline & $\begin{array}{l}\text { Full sample } \\
(N=146)\end{array}$ & $\begin{array}{l}\text { Plasma subsample } \\
(N=59)\end{array}$ \\
\hline Female sex, $\%$ & 63.5 & 65.8 \\
\hline Age, years & $34 \pm 6$ & $34 \pm 6$ \\
\hline $\mathrm{BMI}, \mathrm{kg} / \mathrm{m}^{2}$ & $30 \pm 7$ & $30 \pm 8$ \\
\hline Underweight, $n$ (\%) & $1(0.7)$ & $1(1.7)$ \\
\hline Normal weight, $n(\%)$ & $33(22.6)$ & $12(20.3)$ \\
\hline Overweight, $n(\%)$ & $58(39.7)$ & $24(40.7)$ \\
\hline Obese, $n(\%)$ & $54(37.0)$ & $22(37.3)$ \\
\hline Total water ${ }^{\mathrm{a}, \mathrm{b}} \mathrm{e}, \mathrm{g}$ g/day & $2,640 \pm 1,090$ & $2,354 \pm 939$ \\
\hline Total fiber ${ }^{a, e}, \mathrm{~g} /$ day & $21 \pm 10$ & $20 \pm 9$ \\
\hline Normalized fiber (g/1,000 kcal) & $9.84 \pm 4.46$ & $9.32 \pm 2.85$ \\
\hline$F: B^{b}$ & $2.42 \pm 2.21$ & $2.18 \pm 1.20$ \\
\hline $\mathrm{FMU}_{\mathrm{sg}} \mathrm{a}^{\mathrm{a}, \mathrm{c}} \mathrm{c}$ & $1.018 \pm 0.007$ & $1.019 \pm 0.007$ \\
\hline $\mathrm{FMU}_{\mathrm{vol}}^{\mathrm{b}, \mathrm{c}}, \mathrm{mL}$ & $255 \pm 111$ & $264 \pm 118$ \\
\hline Plasma LBPd, ${ }^{\prime}, \mu g / m L$ & $5.34 \pm 5.69$ & $6.11 \pm 6.29$ \\
\hline Plasma copeptind, $\mathrm{g}, \mathrm{ng} / \mathrm{mL}$ & $1.17 \pm 0.24$ & $1.18 \pm 0.24$ \\
\hline
\end{tabular}

$\mathrm{BMI}$, body mass index; $\mathrm{F}: \mathrm{B}$, Firmicutes to Bacteroides ratio; $\mathrm{FMU}_{\mathrm{sg}}$ first-morning urine specific gravity; $\mathrm{FMU}_{\mathrm{vol}}$, first-morning urine volume; LBP, lipopolysaccharide-binding protein. Significant difference $(p<0.05)$ between variables when split by their respective medians are noted with the superscripts. a Total Water Intake, ${ }^{b} \mathrm{FMU}_{\mathrm{sg},}{ }^{\mathrm{c}} \mathrm{FMU}_{\mathrm{vol},}{ }^{\mathrm{d}}$ Copeptin, e Sex. A subsample of participants provided plasma samples, in which analyses were conducted for LBP, in ${ }^{\mathrm{f}} 95$ participants and copeptin in ${ }^{9} 85$ participants.

median group sizes can be found in the supplementary materials (online suppl. Table 1; for all online suppl. material, see www.karger.com/doi/10.1159/000520478).

\section{Pearson Partial Correlations}

When controlling for age, sex, BMI, and normalized dietary fiber; $\mathrm{FMU}_{\mathrm{sg}}$ was negatively correlated with total water $(r=-0.18, p=0.03)$ and $\mathrm{FMU}_{\mathrm{vol}}(r=-0.26, p=$ $0.002)$ and had a trend level relationship with F:B $(r=$ $0.16, p=0.06)$. There was also a trending relationship between total water and $\mathrm{FMU}_{\mathrm{vol}}(r=0.16, p=0.06)$, but no other significant relationships were found between these variables.

\section{Microbiota Analyses}

Alpha and Beta Diversity Analysis

Alpha diversity was analyzed for within-sample richness using Observed ASVs, Chao1, and ACE richness estimators; and within-sample diversity using Shannon and Simpson diversity indices. These analyses revealed a higher degree of microbial richness in the above median $\mathrm{FMU}_{\mathrm{vol}}$ indicated by observed ASVs $(p=0.03)$, Chaol
Table 2. $p$ Values for above/below median group comparisons of alpha diversity and beta diversity across hydration variables

\begin{tabular}{lllll}
\hline Diversity metric & $\begin{array}{l}\text { Total } \\
\text { water }\end{array}$ & $\mathrm{FMU}_{\mathrm{sg}}$ & $\mathrm{FMU}_{\mathrm{vol}}$ & Copeptin \\
& & & & \\
\hline Alpha diversity & 0.12 & 0.11 & $0.03^{*}$ & 0.65 \\
$\quad$ Observed ASVs & 0.12 & 0.12 & $0.03^{*}$ & 0.65 \\
$\quad$ Chao1 & 0.12 & 0.12 & $0.03^{*}$ & 0.66 \\
$\quad$ ACE & 0.24 & 0.26 & 0.12 & 0.37 \\
$\quad$ Shannon & 0.51 & 0.73 & 0.10 & 0.37 \\
$\quad$ Simpson & & & & \\
Beta diversity & 0.19 & 0.85 & 0.99 & 0.53 \\
$\quad$ Weighted uniFrac & 0.23 & 0.65 & 0.62 & 0.52 \\
$\quad$ Unweighted uniFrac & 0.23 & \\
\hline
\end{tabular}

Alpha diversity metrics were analyzed via Wilcoxon rank-sum test with a holm $p$ value adjustment. Beta diversity metrics were analyzed via PERMANOVA and $p$ values are presented for the respective models. ASV, amplicon sequence variant; $\mathrm{FMU}_{\mathrm{sg}}$, firstmorning urine specific gravity; $\mathrm{FMU}_{\mathrm{vol}}$, first-morning urine volume; PERMANOVA, permutational multivariate analysis of variance. ${ }^{*} p<$ 0.05 .

( $p=0.03)$, and ACE $(p=0.03)$. There were no significant differences between median groups when split by $\mathrm{FMU}_{\mathrm{sg}}$, total water, or copeptin (Table 2).

Beta diversity between groups was analyzed using weighted and unweighted UniFrac matrices [19]. We observed no statistically significant differences in beta diversity between above/below median groups (total water; unweighted $p=0.23$, weighted $p=0.19$ : $\mathrm{FMU}_{\mathrm{sg}}$; unweighted $p=0.65$, weighted $p=0.85: \mathrm{FMU}_{\mathrm{vol}}$; unweighted $p=0.62$, weighted $p=0.99$ : copeptin; unweighted $p=$ 0.52 , weighted $p=0.53$ ) (Table 2).

\section{Linear Discriminant Analysis Effect Size}

LEfSe was conducted to assess differentially abundant taxa based on median splits for total water, $\mathrm{FMU}_{\mathrm{sg}}$, and $\mathrm{FMU}_{\mathrm{vol}}$ independently. There were 37, 60, and $22 \mathrm{ASV}$ s that were differentially abundant, respectively. Several of these features were redundant (i.e., the class, order, and family for a statistically significant genus were also significant); thus, we chose to include only taxa at the genus or species level in the discussion. This reduced the list of differentially abundant taxa to 24 (total water), 35 $\left(\mathrm{FMU}_{\mathrm{sg}}\right)$, and $14\left(\mathrm{FMU}_{\mathrm{vol}}\right)$ unique taxa. LEfSe analyses were then conducted on the copeptin subsample and revealed 23 unique taxa at the genus or species levels. The 10 genera with the highest LDA scores are presented in Figure 2. A full listing of the LEfSe output for each vari-
40

Ann Nutr Metab 2021;77(suppl 4):37-45 DOI: $10.1159 / 000520478$
Willis/Muñoz/Mysonhimer/Edwards/ Wolf/Hillman/Burd/Holscher/Khan 
Fig. 2. Top 10 LEfSe LDA scores of fecal taxa at the genus or species level in groups based on median splits of total water intake

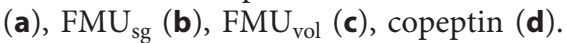
The score indicates differential abundance of taxa and statistically significant magnitude of effect size (at $\alpha=0.05$ ) to the difference between groups. The bars are shaded according to the above (light) or below (dark) median group in which individual taxa were found at greater relative abundance. $F M U_{s g}$, first-morning urine specific gravity; $\mathrm{FMU}_{\mathrm{vol}}$, first-morning urine volume; LEfSe, linear discriminant analysis effect size.

Hydration Biomarkers Are Related to Fecal Microbiota and LBP

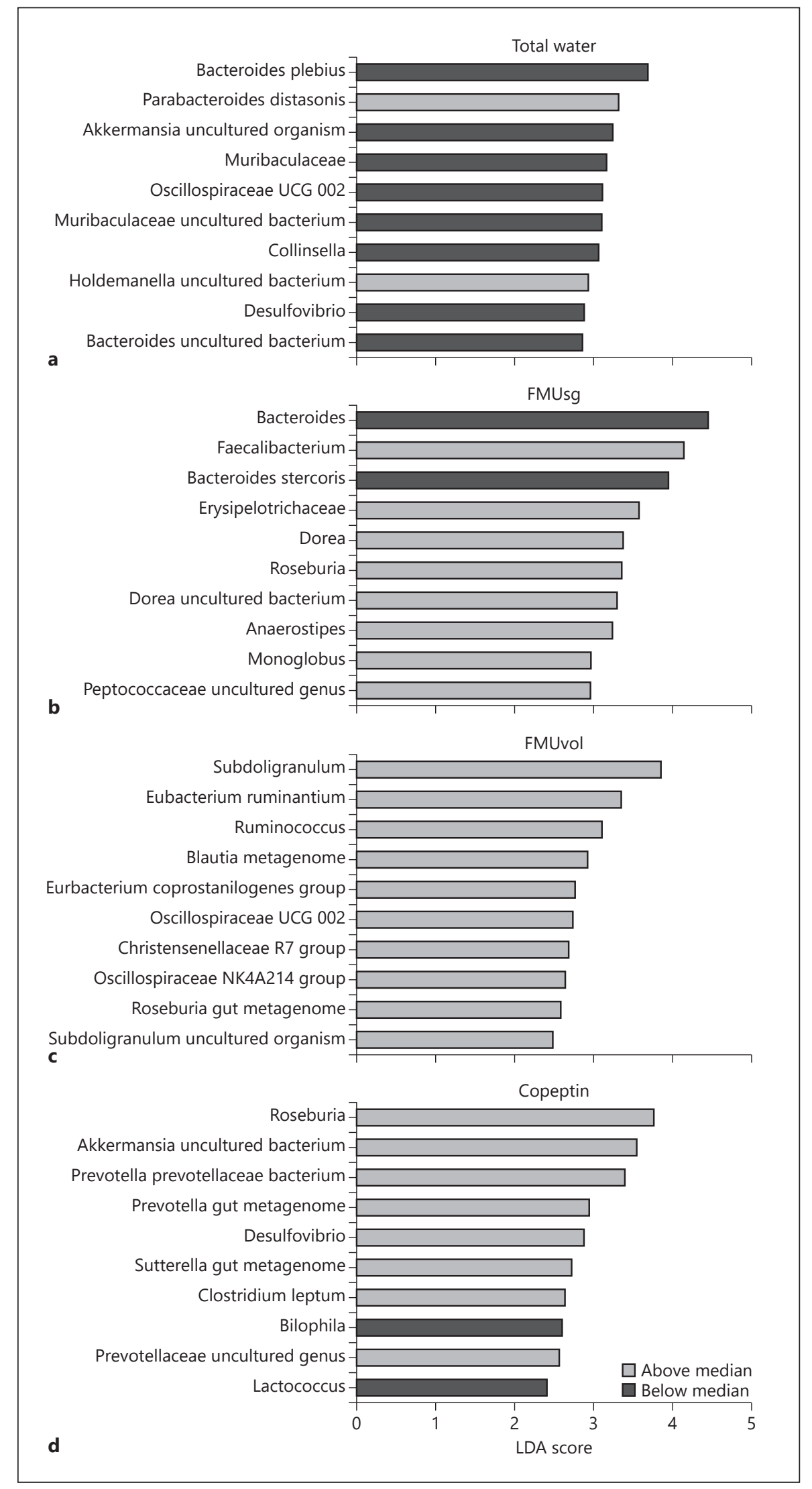

Ann Nutr Metab 2021;77(suppl 4):37-45 


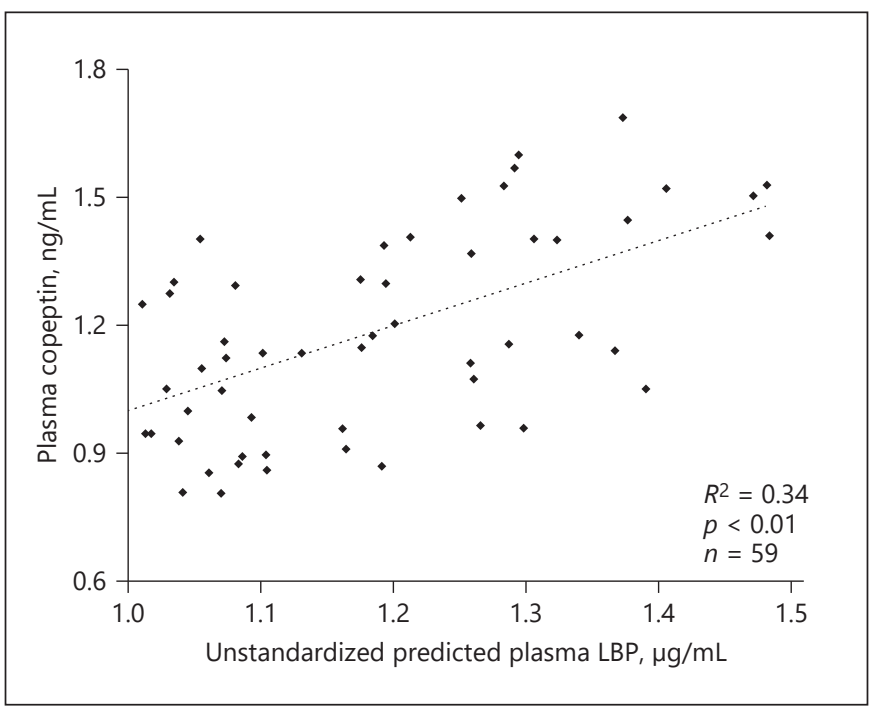

Fig. 3. Scatter plot of copeptin regressed over predicted plasma LBP (controlling for age, sex, BMI, total water, and normalized dietary fiber). Unstandardized residuals from the multiple regression models were used for this plot. LBP, lipopolysaccharide-binding protein; BMI, body mass index.

able may be found in the online supplementary materials (online suppl. Tables 2-5).

\section{Copeptin and LPS Binding Protein}

A regression model comprised of the covariates age, sex, BMI, total water, and normalized dietary fiber explained a significant amount of variance in plasma copeptin values $\left(F(5,53)=2.65, p=0.03, R^{2}=0.20\right)$. Addition of LBP to the model explained an additional $14 \%$ of the variance in copeptin $\left(F(6,52)=4.45, p=0.002, R^{2}=\right.$ 0.34 ; LBP $\beta=0.018, p=0.002$ ) (Fig. 3).

\section{Discussion}

This study examined relations between hydration biomarkers and fecal microbiota. Uncultured species of several bacterial genera were differentially abundant across median $\mathrm{FMU}_{\mathrm{sg}}$, plasma copeptin, and total water. Further, plasma copeptin explained variance in a marker of gut barrier dysfunction as evidenced by a statistically significant association with circulating LBP. To our knowledge, these findings are the first to characterize relations between hydration biomarkers and fecal microbiota and link copeptin to LBP in humans. Uncultured species in the Bacteroides, Desulfovibrio, Roseburia, Peptococcus, and Akkermansia genera were found at greater relative abundance in groups that might indicate poorer hydration status (Table 3 ).

The Bacteroides genus has been a popular target for analysis with a great deal of work specifically examining the polysaccharide degradation capabilities of $B$. thetaiotamicron. These bacteria express enzymes that coordinate the breakdown of multiple specific glycans on the human intestinal epithelium. In fact, $18 \%$ of the $B$. thetaiotamicron genome is dedicated to glycan degradation, evidenced by the discovery of 88 individual polysaccharide utilization loci's in the genome [20]. There is a known relationship between these metabolic generalists and $\mathrm{mu}-$ cin-specialists like Akkermansia muciniphila and Bacteroides caccae such that when dietary fiber is scarce, $A$. muciniphila and B. caccae increase in abundance, cleaving mucin glycans and presumably providing sugar residues for other microorganisms [21]. Interestingly, we observed species in the Akkermansia genus at greater abundance in those with below-median total water intake and above-median copeptin (Table 2). Recently, A. muciniphila has been shown to have improved probiotic traits in response to mucin depletion, inducing mucin secretion and improving barrier function in mice [22]. Roseburia also colonizes the luminal mucus layer and is known for butyrate production [23]. Further, Roseburia have been found in decreased abundance in persons with obesity and type 2 diabetes mellitus [24]; conditions that are also associated with hypohydation.

Desulfovibrio is unique in this group as a genus of sulfate-reducing bacteria. Sulfate reduction yields hydrogen sulfide which can both positively and negatively impacts mucus layer integrity, depending on concentration [25]. Further, Desulfovibrio c21_c20 has been found at greater relative abundance pre-clinically in male Brattleboro rats with AVP gene deletion, when compared to the heterozygous group [26]. While this finding was sex-specific, it does provide interesting evidence as to the potential extent of AVP-microbiota interactions in the gut.

Why these genera were differentially abundant is not immediately clear and will require further investigation to elucidate causal mechanisms. However, that the taxonomic units in common across these hydration markers were all associated with the intestinal mucus layer implies the potential impact of hydration signaling on intestinal barrier function. Indeed, there is evidence that AVP induces mitogenic signaling in response to epithelial injury, and this cell proliferation is posited to play a role in maintaining or recovering mucus and barrier integrity [27].
Willis/Muñoz/Mysonhimer/Edwards/ Wolf/Hillman/Burd/Holscher/Khan 
These findings are especially interesting considering our multiple regression model showing a moderate positive association between plasma copeptin and LBP concentrations. Circulating LPS leads to metabolic endotoxemia, and LPS could have a causal relationship with both intestinal barrier dysfunction and obesity-induced inflammation [14]. While our sample contained persons with overweight and obesity, participants were otherwise screened for metabolic and digestive disorders that frequently contribute to intestinal barrier dysfunction (e.g., inflammatory bowel disease). That LBP accounted for $14 \%$ of the variance of copeptin in this sample suggests that copeptin could be sensitive to metabolic endotoxemia. We cannot offer causal inferences based on these results; however, these findings suggest a novel line of inquiry into the role of AVP and/or copeptin in the modulation of intestinal barrier function that warrants further investigation.

The lack of statistically significant correlations among copeptin and self-reported water consumption was surprising. Copeptin, as a surrogate marker of AVP, has known relationships with hydration markers [28], is elevated in habitually low water consumers [29], and can be attenuated with increased plain water consumption [28]. Thus, we anticipated a negative relationship between plasma copeptin and total water intake; however, this is not unprecedented since the present investigation was not the first to observe such a relationship [28]. The inclusion of urine osmolality based on 24-h samples would have provided interpretive utility beyond FMU and selfreported dietary water intake.

\section{Limitations and Future Directions}

Given the exploratory nature of these aims, we did not posit a directional hypothesis. Nevertheless, establishing links between fecal microbiota and hydration biomarkers serves as a necessary first step in conducting larger studies examining the effects of hydration practices on gastrointestinal and metabolic health. While this study provides novel results linking hydration markers to fecal microbial profiles and plasma LBP, several limitations are worth considering. This was a cross-sectional analysis, and intervention studies are needed to investigate the causal effects of water consumption on gastrointestinal microbiota and barrier integrity. Body water turnover is complex, and ideal biomarkers are context dependent [30]; thus a gold-standard hydration biomarker has yet to emerge. As such, we are unable to make normative claims regarding hydration status based on FMU values alone, though previous work suggests an average $\mathrm{FMU}_{\mathrm{sg}}$ of 1.018 (Table 1)

Hydration Biomarkers Are Related to Fecal Microbiota and LBP

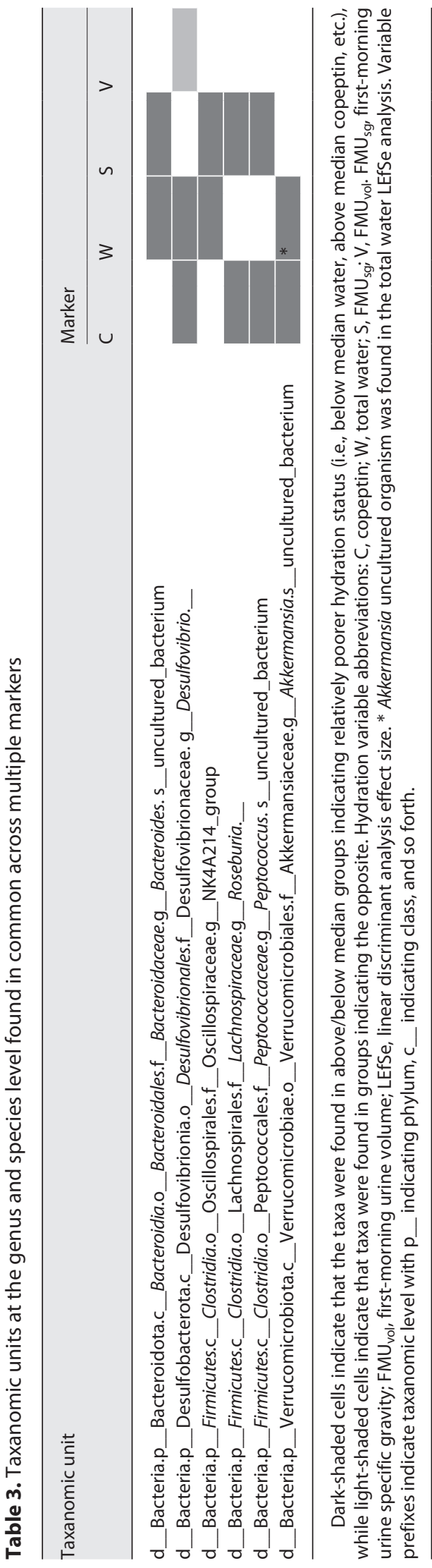

Ann Nutr Metab 2021;77(suppl 4):37-45 DOI: $10.1159 / 000520478$ 
falls between 1.2 and $2.0 \mathrm{~L}$ of daily plain water consumption [7]. Continued work should examine both the relationships between additional biomarkers of hydration (e.g., 24 h urine osmolality), plain water consumption, and the intestinal microbiota; while also considering other confounding factors (e.g., habitual physical activity) to better inform water intake recommendations and public health initiatives.

\section{Acknowledgments}

Special thanks to the research assistants and laboratory technicians in the Body Composition and Nutritional Neuroscience, $\mathrm{Nu}$ trition and Human Microbiome, and Nutrition and Exercise Performance research laboratories for their help with data collection and databasing.

\section{Statement of Ethics}

Primary study protocols were conducted according to the Declaration of Helsinki, and all procedures were approved by the Institutional Review Board of the University of Illinois (Protocol numbers 16840, 16277, 16071). All participants provided written consent before enrollment which included consent to secondary analyses of these data.

\section{Conflict of Interest Statement}

The authors declare no conflicts of interest.

\section{Funding Sources}

This work was supported by the Department of Kinesiology and Community Health and the Division of Nutritional Sciences at the University of Illinois at Urbana-Champaign, the USDA National Institute of Food and Agriculture, Hatch project 1009249, the Hass Avocado Board (Institutional Award Number 079273), and the Cancer Education and Career Development Program (T32CA057699).

\section{Author Contributions}

The followings are the authors' contributions: conceptualization: N.B.W. and N.A.K.; formal analysis: N.B.W. and A.R.M.; funding acquisition: H.D.H. and N.A.K.; investigation: C.G.E. and N.A.B.; writing - original draft: N.B.W., C.X.M., and N.A.K.; writing - review and editing: A.R.M., C.G.E., P.W., C.H.H., N.A.B., and H.D.H.

\section{Data Availability Statement}

All data generated or analysed during this study are included in this article and its online supplementary files. Further enquiries can be directed to the corresponding author.

\section{References}

1 Kavouras SA, Anastasiou CA. Water physiology. Nutr Today. 2010;45(6):S27-32.

2 Perrier ET. Shifting focus: from hydration for performance to hydration for health. Ann Nutr Metab. 2017;70(Suppl 1):4-12.

3 Otten JJ, Hellwig JP, Linda D. Dietary reference intakes. Washington, DC: National Academies Press; 2006.

4 Stookey JD. Analysis of 2009-2012 nutrition health and examination survey (NHANES) data to estimate the median water intake associated with meeting hydration criteria for individuals aged $12-80$ in the US population. Nutrients. 2019;11(3):657.

5 Stookey JD, Kavouras SA, Suh H, Lang F. Underhydration is associated with obesity, chronic diseases, and death within 3 to 6 years in the U.S. population aged $51-70$ years. $\mathrm{Nu}-$ trients. 2020;12(4):905

6 Perrier ET, Buendia-Jimenez I, Vecchio M, Armstrong LE, Tack I, Klein A. Twenty-fourhour urine osmolality as a physiological index of adequate water intake. Dis Markers. 2015; 2015:231063

7 Perrier E, Vergne S, Klein A, Poupin M, Rondeau P, Le Bellego L, et al. Hydration biomarkers in free-living adults with different levels of habitual fluid consumption. Br J Nutr. 2013;109(9):1678-87.

8 Morgenthaler NG, Struck J, Jochberger S, Dünser MW. Copeptin: clinical use of a new biomarker. Trends Endocrinol Metab. 2008; 19(2):43-9.

9 Keppens S, De Wulf H. The nature of the hepatic receptors involved in vasopressin-induced glycogenolysis. Biochim Biophys Acta. 1979;588(1):63-9.

10 Monstein HJ, Truedsson M, Ryberg A, Ohlsson B. Vasopressin receptor mRNA expression in the human gastrointestinal tract. Eur Surg Res. 2008;40(1):34-40.

11 Müller B, Morgenthaler N, Stolz D, Schuetz P, Müller C, Bingisser R, et al. Circulating levels of copeptin, a novel biomarker, in lower respiratory tract infections. Eur J Clin Invest. 2007; 37(2):145-52

12 Enhörning S, Bankir L, Bouby N, Struck J, Hedblad B, Persson M, et al. Copeptin, a marker of vasopressin, in abdominal obesity, diabetes and microalbuminuria: the prospective Malmö Diet and Cancer Study cardiovascular cohort. Int J Obes. 2013;37(4):598-603.

13 Fuke N, Nagata N, Suganuma H, Ota T. Regulation of gut microbiota and metabolic endo- toxemia with dietary factors. Nutrients. 2019; 11(10):2277.

14 Cani PD, Amar J, Iglesias MA, Poggi M, Knauf C, Bastelica D, et al. Metabolic endotoxemia initiates obesity and insulin resistance. Diabetes. 2007;56(7):1761-72.

15 Vreugdenhil AC, Rousseau CH, Hartung T, Greve JW, van 't Veer C, Buurman WA. Lipopolysaccharide (LPS)-binding protein mediates LPS detoxification by chylomicrons. J Immunol. 2003;170(3):1399-405.

16 Callahan BJ, McMurdie PJ, Rosen MJ, Han AW, Johnson AJ, Holmes SP. DADA2: highresolution sample inference from Illumina amplicon data. Nat Methods. 2016;13(7):581-3.

17 Bolyen E, Rideout JR, Dillon MR, Bokulich NA, Abnet CC, Al-Ghalith GA, et al. Reproducible, interactive, scalable and extensible microbiome data science using QIIME 2. Nat Biotechnol. 2019;37(8):852-7.

18 Holscher HD, Guetterman HM, Swanson KS An R, Matthan NR, Lichtenstein AH, et al. Walnut consumption alters the gastrointestinal microbiota, microbially derived secondary bile acids, and health markers in healthy adults: a randomized controlled trial. J Nutr. 2018;148(6):861-7. 
19 Lozupone CA, Hamady M, Kelley ST, Knight R. Quantitative and qualitative beta diversity measures lead to different insights into factors that structure microbial communities. Appl Environ Microbiol. 2007;73(5):1576-85.

20 Martens EC, Chiang HC, Gordon JI. Mucosal glycan foraging enhances fitness and transmission of a saccharolytic human gut bacterial symbiont. Cell Host Microbe. 2008;4(5): 447-57.

21 Desai MS, Seekatz AM, Koropatkin NM, Kamada N, Hickey CA, Wolter M, et al. A dietary fiber-deprived gut microbiota degrades the colonic mucus barrier and enhances pathogen susceptibility. Cell. 2016;167(5):1339-53. e21.

22 Shin J, Noh J-R, Chang D-H, Kim Y-H, Kim $\mathrm{MH}$, Lee ES, et al. Elucidation of Akkermansia muciniphila probiotic traits driven by mucin depletion. Front Microbiol. 2019;10:1-12.
23 Van Den Abbeele P, Belzer C, Goossens M, Kleerebezem M, De Vos WM, Thas O, et al. Butyrate-producing Clostridium cluster XIVa species specifically colonize mucins in an in vitro gut model. ISME J. 2013;7(5):94961.

24 Barlow GM, Yu A, Mathur R. Role of the gut microbiome in obesity and diabetes mellitus. Nutr Clin Pract. 2015;30(6):787-97.

25 Blachier F, Beaumont M, Kim E. Cysteinederived hydrogen sulfide and gut health: a matter of endogenous or bacterial origin. Curr Opin Clin Nutr Metab Care. 2019;22(1): 68-75.

26 Fields CT, Chassaing B, Paul MJ, Gewirtz AT, de Vries GJ. Vasopressin deletion is associated with sex-specific shifts in the gut microbiome. Gut Microbes. 2018;9(1):13-25.
27 Chiu T, Wu SS, Santiskulvong C, Tangkijvanich P, Yee HF, Rozengurt E. Vasopressinmediated mitogenic signaling in intestinal epithelial cells. Am J Physiol Cell Physiol. 2002;282(3):C434-50.

28 Lemetais G, Melander O, Vecchio M, Bottin $\mathrm{JH}$, Enhörning S, Perrier ET. Effect of increased water intake on plasma copeptin in healthy adults. Eur J Nutr. 2018;57(5):1883.

29 Perrier E, Demazières A, Girard N, Pross N, Osbild D, Metzger D, et al. Circadian variation and responsiveness of hydration biomarkers to changes in daily water intake. Eur J Appl Physiol. 2013;113(8):2143-51.

30 Muñoz CX, McKenzie AL, Armstrong LE. Optimal hydration biomarkers: consideration of daily activities. Obes Facts. 2014;7(2): 13-8. 